\title{
Tratamiento de la microforma labial
}

\author{
Repair of microform cleft lip
}

Monasterio Aljaro, L.*

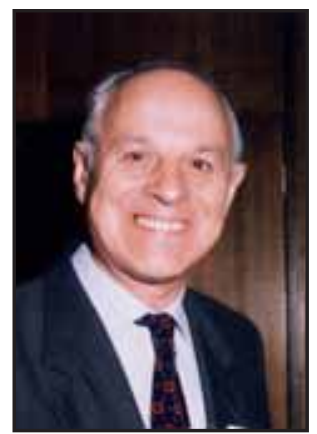

Monasterio Aljaro, L.
Resumen

La microforma labial es un tipo de fisura labial muy poco frecuente, que corresponde solo al $1 \%$ de los pacientes con hendidura labial unilateral. Las características anatómicas más importantes de esta anomalía son: alteración de la línea cutáneo-mucosa a nivel del arco de Cupido, surco en el rojo labial, disrupción del músculo orbicular y malformación nasal menor. Se clasifican en unilaterales y bilaterales.

Realizamos un estudio retrospectivo sobre 51 casos controlados en la Fundación Gantz ( Santiago de Chile) en los últimos 10 años. Exponemos con detalle y resultados ilustrados una técnica personal que empleamos desde 2005, que resuelve el problema labial y nasal mediante incisiones mínimas y comentamos otras técnicas publicadas al respecto.
Palabras clave Fisura labial, Microforma labial.

Código numérico 2312-23122
Key words $\quad$ Cleft lip, Microform cleft lip.

Numeral Code $\quad 2312-23122$
Abstract

Microform cleft lip is an unusual pathology (1\% of patients with unilateral cleft lip) characterized by: notched mucosal margin in the Cupid bow, cleft in red lip, abnormality in the orbicularis oris and minor nasal deformity.

We present a retrospective study done in Fundación Gantz (Santiago de Chile) during last 10 years, based on 51 cases operated on with a personal technique used since 2005, that solves nasal and lip abnormalities with minimal incisions. We present technical details and illustrated cases an a review of the published literature about the theme. 


\section{Introducción}

Las fisuras faciales comprenden un amplio espectro en lo que se refiere a intensidad, localización y conductas terapéuticas. En un extremo del abanico de las fisuras unilaterales se encuentra las fisuras que tiene más compromiso y que incluyen labio y paladar y en el otro las llamada microformas (Fig. 1). En la literatura reciben diversos nombres: microfisura, minifisura, fisura tipo cicatriz congénita, fisura labial oculta, fisura frustra, fisura labial subcutánea, sin embargo el termino de microforma labial es el más recomendado. Pueden ser de tipo unilateral (las más frecuentes) o bilaterales, que a su vez pueden ser, simétricas (muy raras) o asimétricas, más comunes (Fig. 2, 3).

Por presentar una anomalía de carácter leve o poco llamativa, estos pacientes habitualmente o no consultan o lo hacen tardíamente, llegando muchos de ellos a la edad adulta sin tratamiento

Las alteraciones anatómicas más importantes que presentan son (Fig. 3): irregularidad en el borde mucoso marginal con la presencia de un surco en el rojo labial, menor desarrollo del segmento medial del bermellón, interrupción de la línea cutáneo mucosa a nivel del pico del arco de Cupido, músculo orbicular alterado por falta de unión, presencia de una banda fibrosa cutánea desde el rojo labial hasta el piso nasal dispuesta verticalmente a lo largo de la columna filtral (a veces con algún grado de pigmentación de la piel), alteración de la nariz con asimetría de las ventanas nasales, piso nasal más amplio de lo normal y cartílago alar descendido. Pueden asociarse a anomalías dento-alveolares con déficit óseo en la escotadura piriforme y el incisivo lateral alterado en su posición, forma y tamaño. Estas anomalías no siempre se presentan todas y se pueden combinar entre ellas.

Las causas últimas de esta patología no son conocidas; Cosman (1), señala que seria por una falta de penetración del mesodermo en una envoltura mesodérmica intacta y no por la clásica explicación de fallo en la fusión del proceso nasal medial con el proceso maxilar. Castilla y col.(2) plantean la provocativa idea de que podría tratarse de una fisura labial que cicatrizo en una etapa tardía. Cartens (3) postula la presencia de 4 diferentes "campos embrionarios" y señala que el sitio de la fisura estaría localizado en la interfase del campo A con el B en el interior de la pared piriforme lateral.
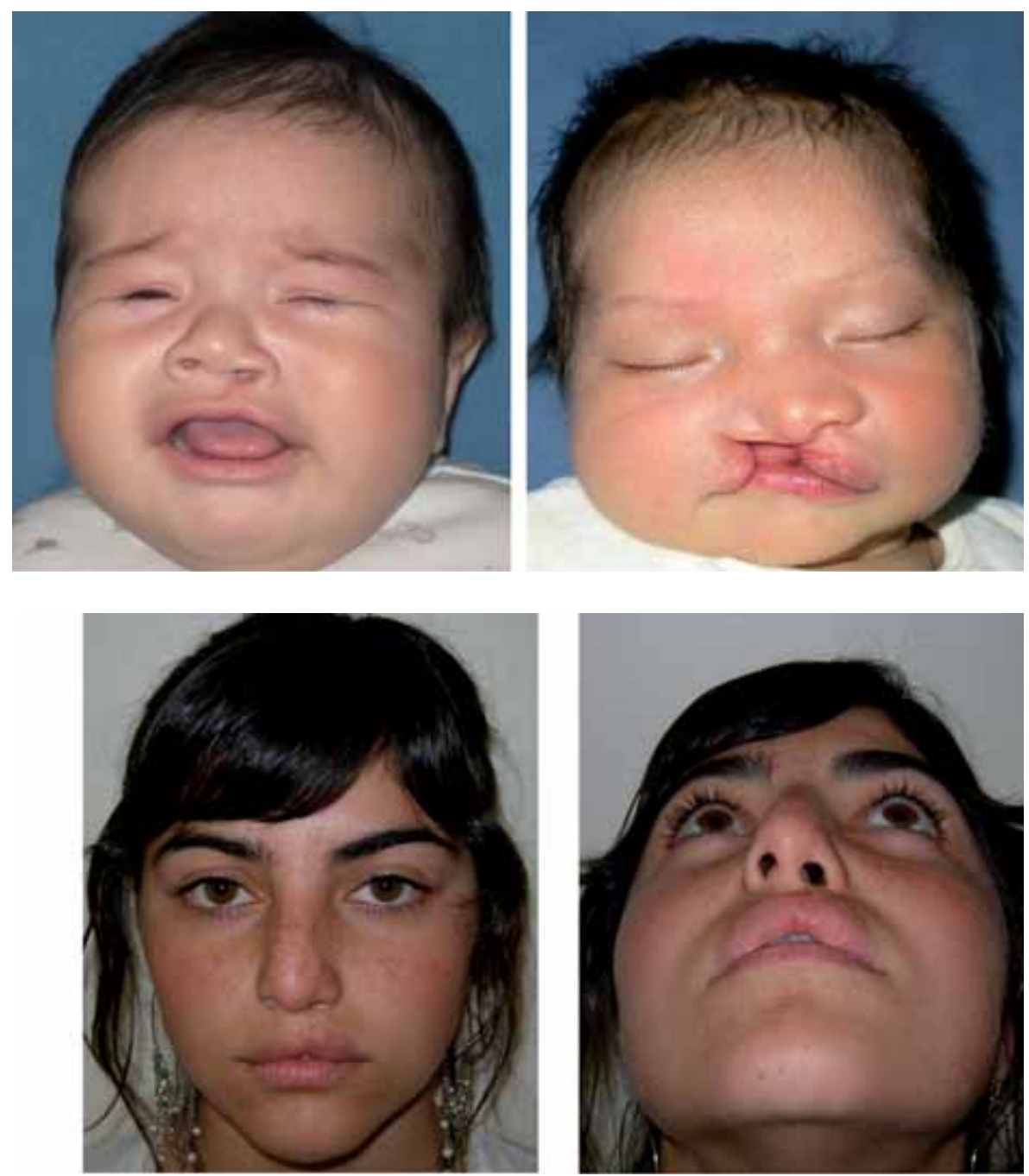

Fig. 2. Fisura unilateral izquierda no tratada en paciente de 14 años; se aprecia la malformación nasal y el aspecto de cicatriz en el labio con un surco en el bermellón.

Fig. 1. Expresiones de la misma patología con diferente intensidad. A la izquierda lactante con Microfisura labial izquierda; a la derecha, con una fisura completa labio-palatina. 

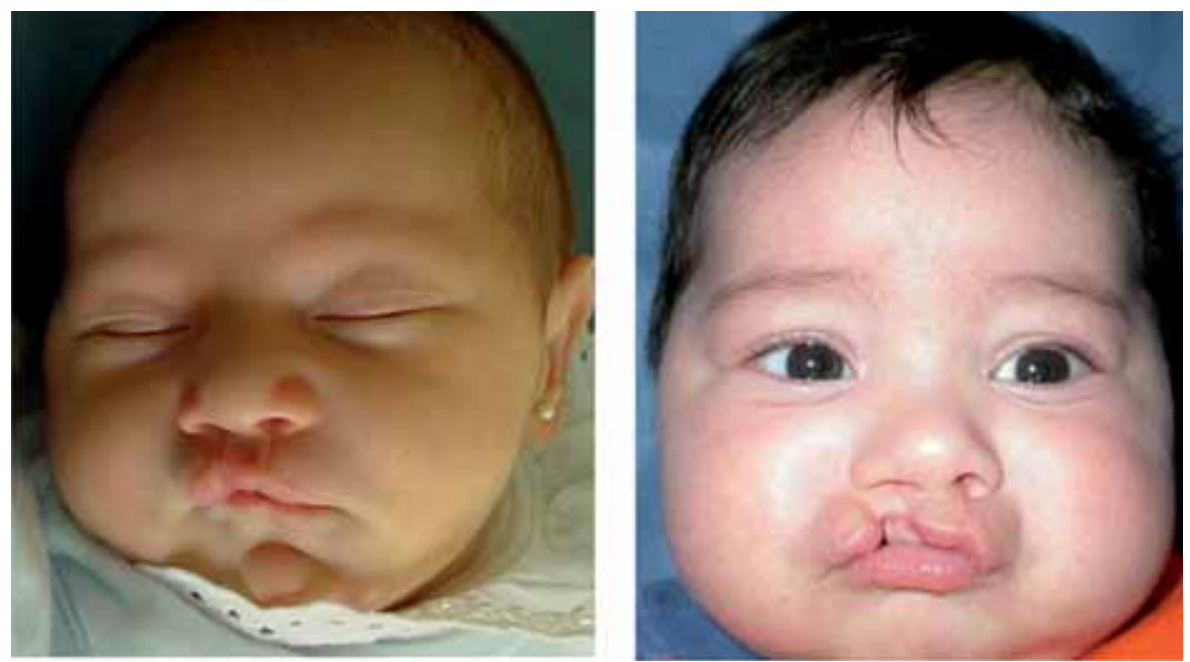

Fig. 3. Fisura bilateral tipo microforma: a la izquierda de tipo simétrico; en el lado derecho otro paciente con una fisura asimétrica, con microfisura izquierda y fisura $2 / 3$ derecha.

La corrección quirúrgica de esta condición ha recibido poco interés en la literatura, posiblemente porque la lesión es poco aparente y la reparación pareciera ser simple; se ha planteado el dilema de corregirla con una completa incisión cutánea o realizar una intervención más limitada mediante incisiones mínimas.

\section{Material y método}

Llevamos a cabo una revisión retrospectiva de los pacientes registrados con este diagnóstico en la Fundación Gantz (Santiago de Chile) en los últimos 10 años consignando: edad de consulta, tipo de lesión, edad y método de tratamiento empleado. La revisión de los registros clínicos, señaló 51 pacientes portadores de algún tipo de Microforma Labial, 32 varones y 19 mujeres. Del tipo unilateral fueron 32 casos (22 izquierdo, 10 derecho), bilaterales asimétricos 17 y solo 2 pacientes presentaban fisuras bilaterales simétricas.

\section{Técnica quirúrgica propuesta}

Para la intervención, los pacientes fueron sometidos a anestesia general, con tiempo operatorio medio de 40 minutos.

Comenzamos con la marcación, detallada más adelante, y la infiltración de labio y nariz con una solución vasoconstrictora de epinefrina al 1 por 100.000.
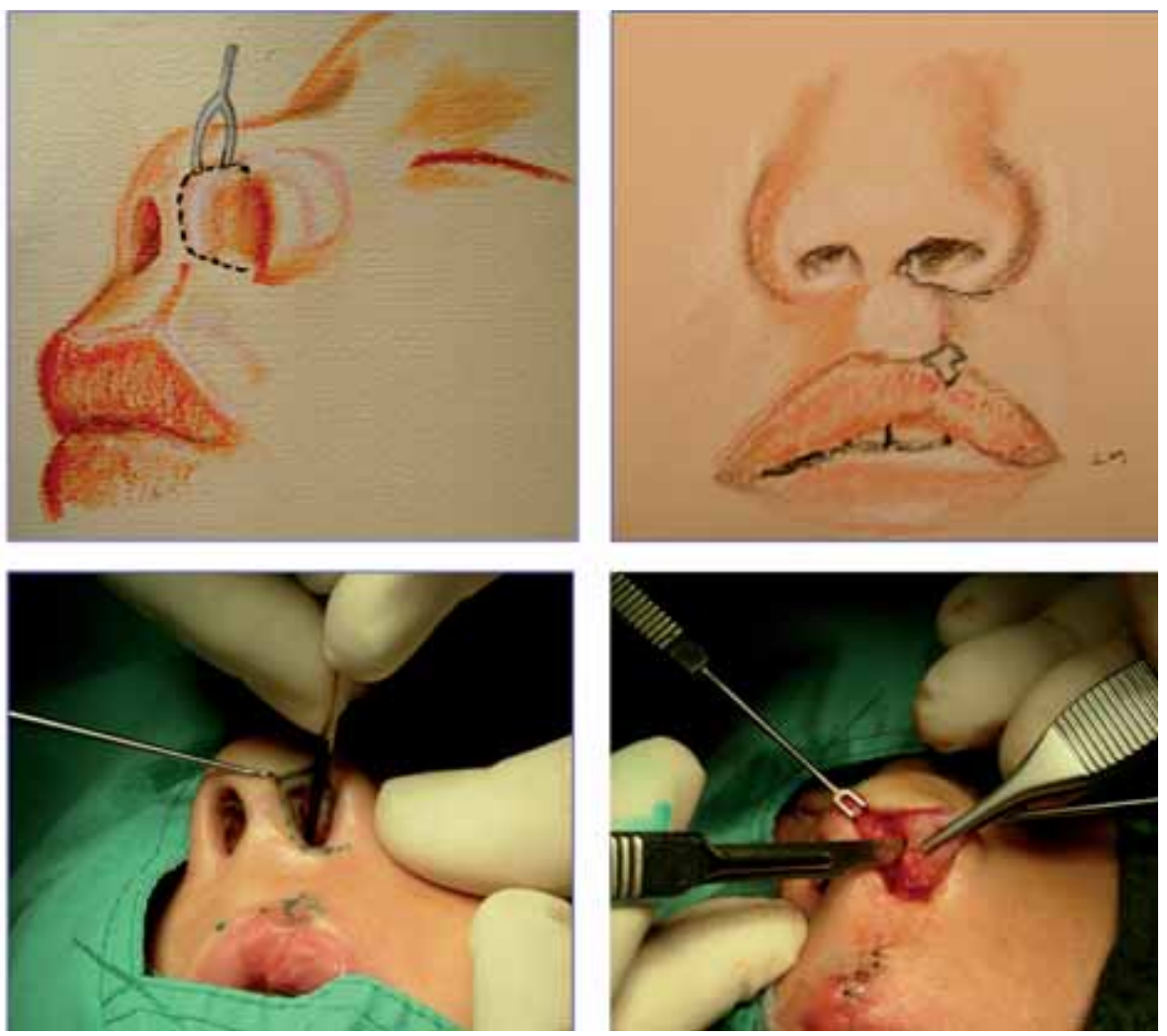

Fig. 4. Marcación de las incisiones: la superior en el piso nasal y borde de la ventana nasal hasta el techo y la inferior en la línea cutáneo-mucosa. En foto inferior derecha se aprecia la liberación del cartílago alar. 

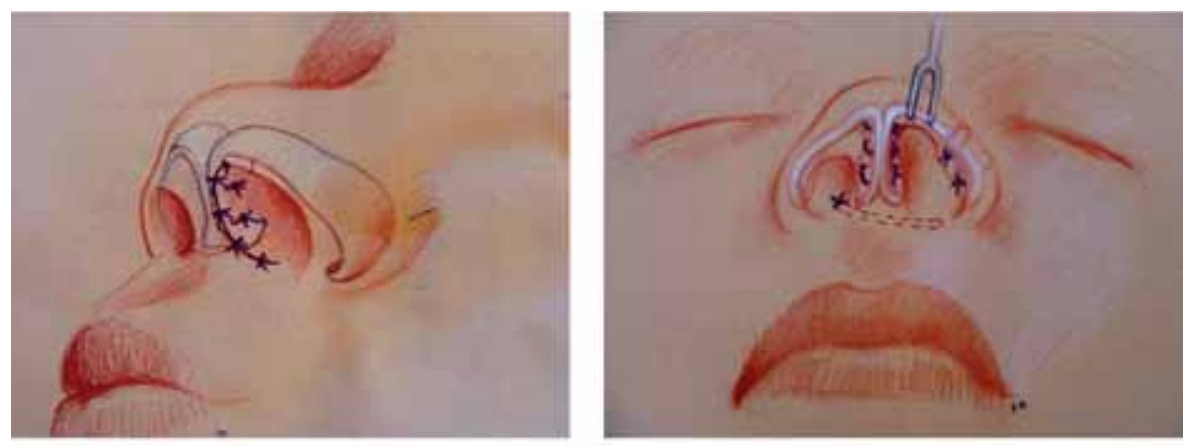

Fig. 5. Fijación de los cartílagos alares mediante varias suturas en U. En las figuras inferiores, paciente en visión basal, pre y postoperatorio.
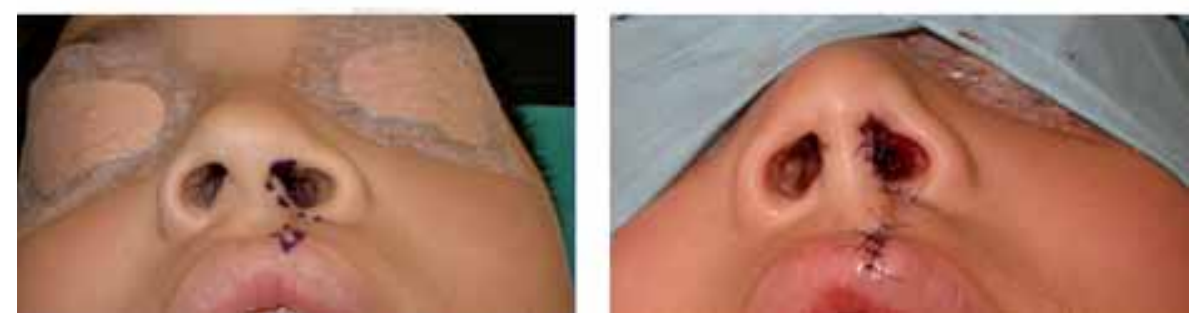

Realizamos sobre la piel en el lado afectado del labio superior, dos marcaciones con verde brillante (Fig.4), una superior, a nivel del piso nasal, que se extiende desde la implantación del ala nasal, a través del piso, luego por el borde medial de la ventana nasal afectada, hasta el techo de la misma y que nos permite abordar el cartílago nasal desplazado, liberándolo de la piel nasal y de la crus medial del cartílago opuesto, para posteriormente, movilizar y fijar el cartílago en la posición correcta con algún grado de sobrecorrección; adicionalmente puede ser necesario realizar una suspensión del cartílago lateral inferior ipsilateral. La fijación de las crus mediales, como se observa en la figura 5, se realiza con puntos transcolumelares en $\mathrm{U}$ empleando material de lenta reabsorción (Monocryl ${ }^{\circledR} 4$ ó 5-0 ); el piso nasal se calibra mediante punto que ancla el músculo transverso nasal a la espina nasal; por otra parte la incisión en el piso nasal permitirá, además del avance medial alar, tener acceso al músculo orbicular que generalmente se presenta con una diástasis o deprimido, para liberarlo subcutáneamente de la piel hasta casi el rojo labial y mediante varios puntos en $\mathrm{U}$, unir sus haces y recrear en alguna medida la cresta filtral (Fig. 6).

La marcación inferior en la línea cutánea (Fig. 4 y 5), se inicia señalando el pico del arco de Cupido del lado sano, el punto medio del arco de Cupido y el punto donde está el vértice del arco de Cupido del lado afecto, que casi siempre está más alto y requiere para su descenso una pequeña incisión de descarga medial. En el borde lateral realizamos el diseño de un triángulo con una base externa de $1 \mathrm{a} 2 \mathrm{~mm}$ de ancho, e inmediatamente por arriba de la línea cutáneomucosa, en la zona correspondiente al vértice del arco de Cupido, triángulo que introducirá en incisión de descarga realizada en el borde medial, resecando la piel que está entre ambas incisiones. De esta manera se obtiene una simetría entre ambos lados, con un arco de Cupido balanceado, puesto que generalmente hay un cierto grado de acortamiento del lado afecto al compararlo con el normal que nos obliga a corregir la deficiencia vertical. Si hubiese un surco en el rojo
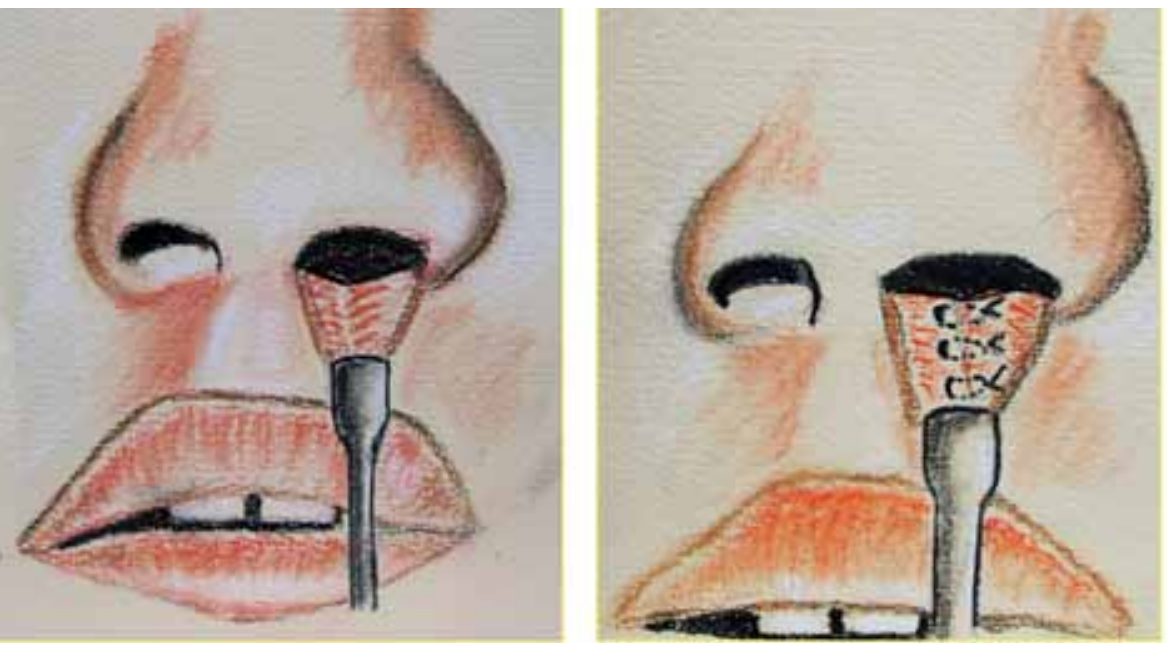

Fig. 6. Sutura del músculo orbicular a través de la incisión en piso nasal, reconstituyendo la continuidad muscular. 


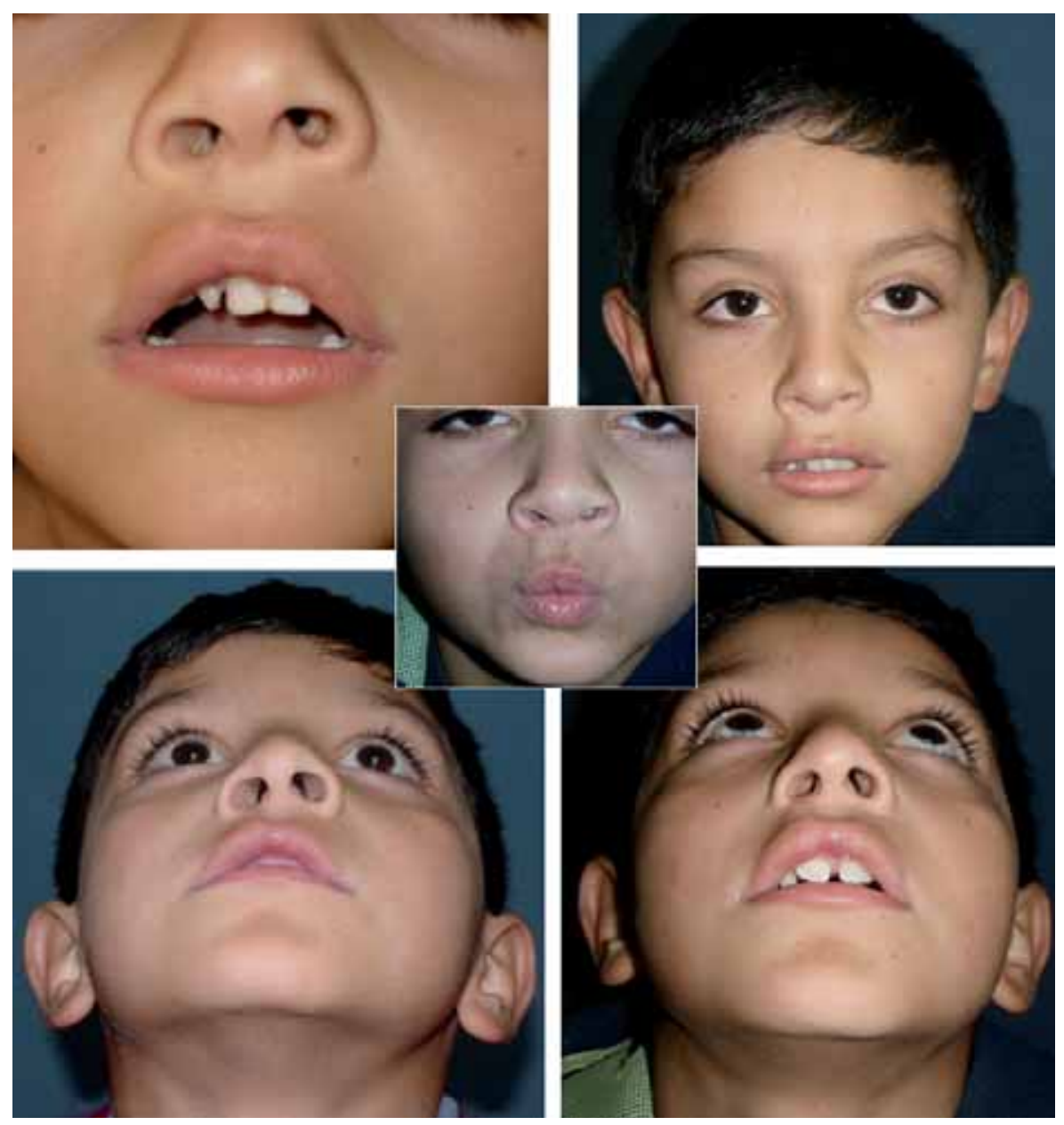

Fig. 7. Caso ilustrativo: a la izquierda, aspecto preoperatorio en un paciente de 6 años; a la derecha 2 meses después de la operación en la que se realizó plastia muscular y nasal y colgajo triangular en la línea cutáneo-mucosa, con resultados estético y funcional satisfactorios, empleando incisiones mínimas.

labial, procedemos a realizar una Z-plastia, o mejor, a intercalar un colgajo triangular de base externa tipo Noordhoff que servirá para resolver el déficit del tubérculo a veces presente; para el cierre de las incisiones cutáneas se emplea Nylon 6-0.

\section{Resultados}

En nuestro centro, los 51 pacientes revisados con este tipo de lesión corresponden al $1.1 \%$ de las fisuras labiales diagnosticadas. La edad de la primera consulta fluctúo entre 1 mes y 42 años. Solo 7 pacientes con fisura unilateral se presentaron en consulta antes de los 3 meses de edad, la mayoría lo hicieron a edades más tardías. Del total de pacientes, en 31 no se había realizado ninguna intervención. En 29 familias, de las cuales uno de los progenitores era portador de fisura labial, y tenían al menos un hijo con fisura labial, hubo 5 casos de microforma unilaterales entre los padres y todos ellos tuvieron hijos con fisuras completas de labio. Desde el año 2006 se emplea la técnica anteriormente descrita, operando 12 pacientes con microforma unilateral. Todos, con resultados satisfactorios desde el punto de vista funcional y estético, sin que se registraran complicaciones; las intervenciones fueron realizadas con hospitalización tran- sitoria, permaneciendo en observación durante 6 horas, siendo luego dados de alta a su domicilio. Se realizó control a las 48 horas y los puntos fueron retirados al quinto día de postoperatorio (Fig. 7-9). El seguimiento de los pacientes fue desde 1 mes a 48 meses, promedio 4 meses.

\section{Discusión}

La microforma labial. debido a su poca notoriedad, es muchas veces ignorada y no diagnosticada oportunamente durante el periodo de recién nacido, por lo que los padres no son informados o reciben información incorrecta, diciéndoles que con el crecimiento se resolverá espontáneamente, lo que por supuesto no es real.

En nuestra revisión, observamos que una minoría de los pacientes concurren a consulta en edades tempranas (de 32 pacientes unilaterales solo 7 consultaron antes de los 3 meses), debido a la naturaleza de la condición, y aun más, muchos llegan hasta la edad adulta sin recibir tratamiento quirúrgico.

Observamos que en familias con múltiples casos, cuando un progenitor presentaba fisura tipo microforma (5 casos), su descendencia presentó un tipo de fisura más severa ( completa del labio) lo que indica- 

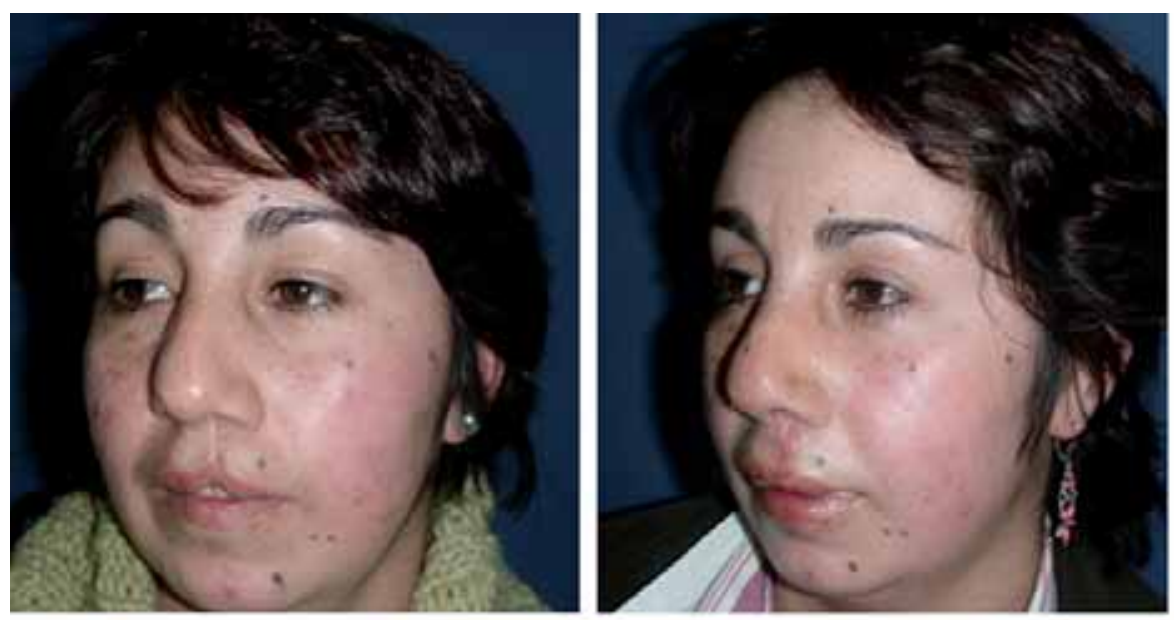

Fig. 8. Paciente de 34 años con microfisura izquierda: a la izquierda antes de la operación y a la derecha 3 meses después de la misma. Nótese la simetría de las ventanas nasales.
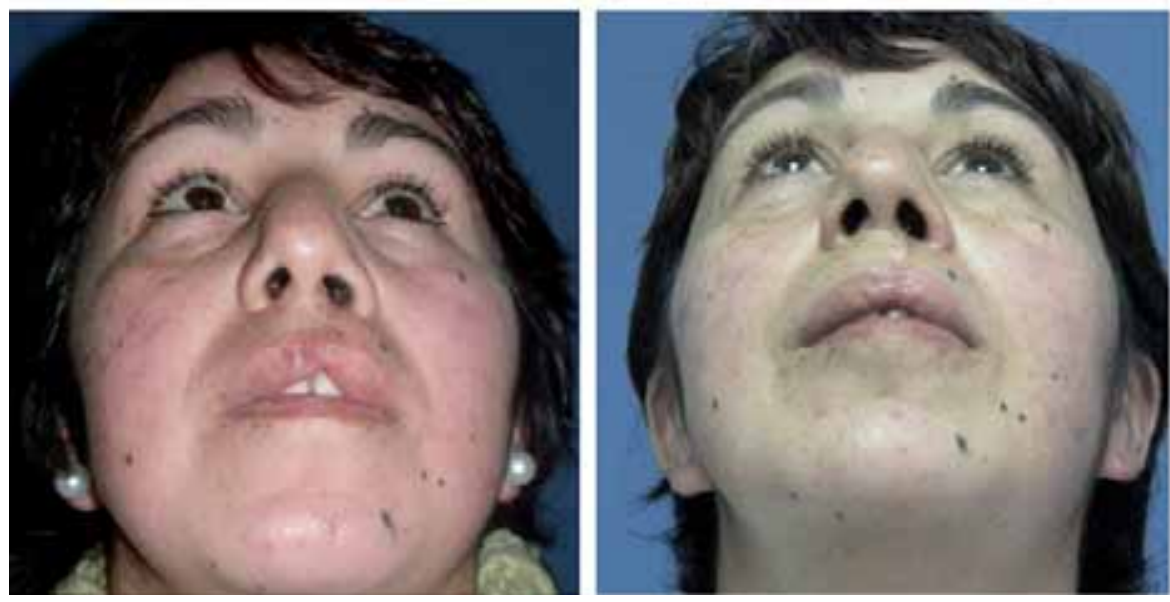

ría que la microforma determina en la descendencia fisuras fenotípicas más intensas.

La mayor incidencia en hombres con respecto a mujeres y en el lado izquierdo más que en el derecho, coincide con los hallazgos descritos en otras publicaciones.

En la literatura médica hay muy pocas referencias en relación a técnicas quirúrgicas aplicables para resolver los problemas que plantea esta anomalía. El tratamiento de estos pacientes dependerá del tipo de lesión y de su intensidad y el objetivo será restaurar la continuidad muscular, regularizar la línea cutáneo mucosa, el surco labial y reparar la anomalía nasal, idealmente, con la menor cicatriz externa visible.

Millard (4), en su monumental obra "Cleft Craft" Tomo 1, presenta un solo caso operado en el cual se
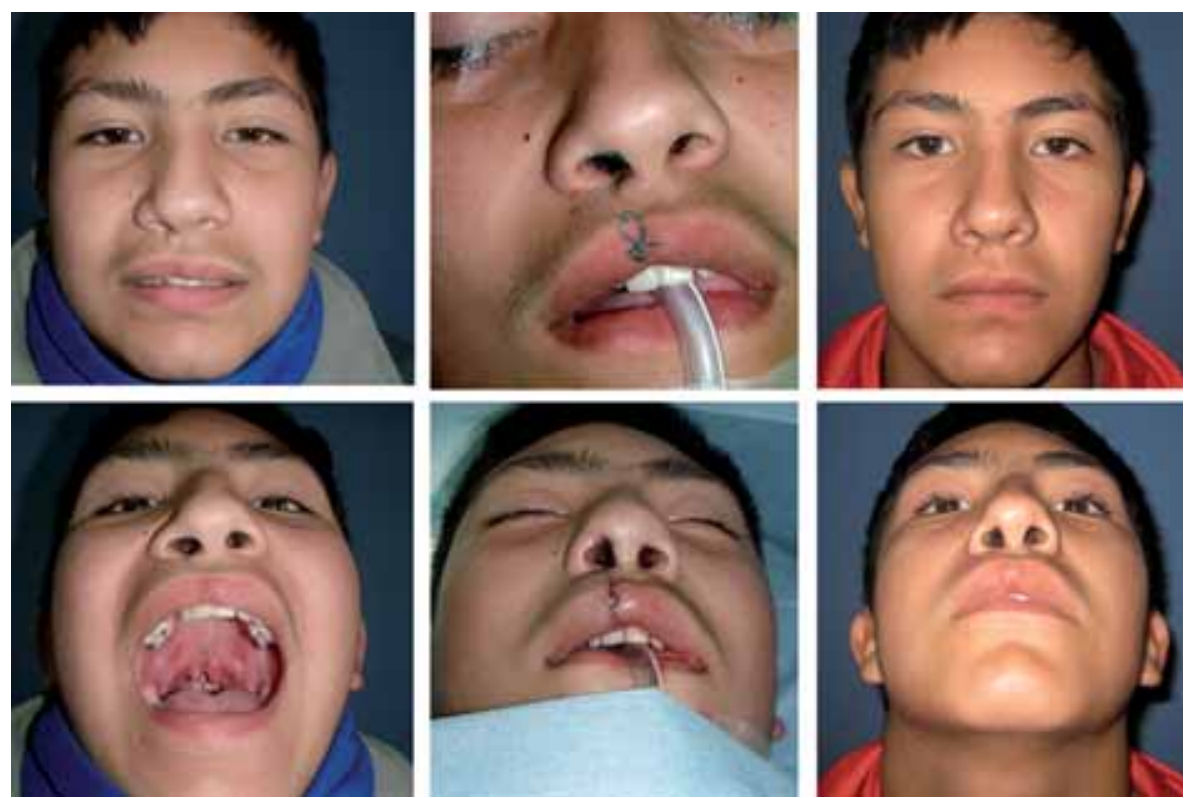

Fig. 9. Paciente de 12 años con microforma labial derecha y fisura submucosa asintomática: a la izquierda antes de la operación, en el centro marcaje y postoperatorio inmediato y a la derecha, 6 meses después de la cirugía. 
incindió completamente la piel y se suturó el músculo orbicular, pero no se hizo rotación y avance de los colgajos, con un excelente resultado final. Thaller (5) utilizó el mismo procedimiento en otro caso. Onizuca y col. (6), en 1991, preconizaron el uso de incisiones mínimas, utilizando una Z-plastia a nivel de la línea cutáneo mucosa, que recrea la columna filtral con un colgajo muscular, y trata la nariz con resección lenticular en el piso y reposición del cartílago alar. Cho (7) publica una técnica realizada en 12 pacientes en la cual emplea una incisión interna a través de la mucosa bucal vestibular, interdigitando el músculo orbicular, Z-plastia en la unión cutáneo mucosa y trata la nariz mediante avance del ala, reposición y fijación del cartílago alar a través de la incisión de Tajima, con excelentes resultados estéticos y funcionales.

Mulliken (8), en una extensa y reciente revisión del tema, propone el uso de una doble Z-plastia atípica (con una pata), para resolver el problema de la línea blanca, del bermellón y del músculo, practicando incisiones mínimas. Incluye, a lo largo de la cresta filtral, un injerto libre de dermis subcutáneo sobre el músculo con el objeto de aumentar la columna filtral. Realiza una reposición del cartílago y base alar. Intervino a 23 pacientes, persistiendo en un tercio de ellos la asimetría nasal, mientras que en un 13\% la columna filtral se aprecia poco prominente.

Nuestro tratamiento hasta el año 2005 consistía en una incisión completa de la piel a lo largo de la lesión y a través de ella se procedía al tratamiento del músculo y a la regularización del arco de Cupido con incisión de todo el rojo labial. Además abordábamos la nariz mediante una incisión marginal, reposicionando el cartílago alar. Si bien lográbamos buenos resultados, corríamos siempre el riesgo de que la calidad de la cicatrización, en algunos pacientes pudiera ser hipertrófica y que el resultado final fuera peor que el defecto inicial. Por este motivo y tras revisar la literatura, empleamos desde hace 3 años este nuevo método que presentamos, con incisiones mínimas, que permite resolver la anomalía muscular, nasal y del ber- mellón con resultados que han sido muy satisfactorios (9). En 2007, durante la Reunión de la ACPA (American Cleft Palate Association) en Denver (EEUU) (10) presentamos los primeros casos.

\section{Conclusiones}

Los pacientes con microforma labial presentan alteraciones de poca intensidad en el labio y en la nariz que plantean diferentes alternativas de tratamiento; el cirujano debe esforzarse, puesto que la distancia con lo forma normal es pequeña y debe realizar una operación que solucione todas las anomalías presentes mediante incisiones mínimas, que determinen cicatrices inaparentes.

\section{Dirección del autor}

Dr. Luis Monasterio Aljaro

Calle Los Laureles 1110, Dep. 1003

Vitacura, Santiago, Chile.

e-mail: fundación@gantz.cl

\section{Bibliografía}

1. Cosman B.et al.: "The minimal cleft lip".Plast.Recontr.Surg. 1968, 41:35.

2. Castilla E. et al.: "Congenital healing cleft lip". Am J. Med .Genet. 1995, 58:106.

3. Cartens M.: "The spectrum of minimal clefting: process oriented cleft management in the presence of an intact alveolus". J. Craniofac. Surg. 2000, 33: 270.

4. Millard R.: "Cleft Craft" Vol.1. The unilateral deformity. Boston: Little Brown 1976.

5. Thaller S. et al.: "Microform cleft lip associated with complete cleft palate". Cleft Palate Craniofac. 1995, 32:247.

6. Onizuca T. et al.: "Operation of microform of cleft lip". Cleft Palate Craniofac. 1991, 28:293.

7. Cho B.C.: "New technique for correction of the microform cleft lip using vertical interdigitation of the orbicularis oris muscle through the intraoral incision". Plas. Recontr. Surg. 2004, 114:1032.

8. Mulliken J.: "Repair Double Unlimb Z-Plastic Repair of Microform Cleft Lip". Plast Recons Surg. 2005, 116:1623.

9. Monasterio L.: "Tratamiento Interdisciplinario de las Fisuras Labio Palatinas". Fundación Gantz. Santiago de Chile,2008.

10. Monasterio L. Ford A.: "Technique for correction of patients with microform cleft lip nose" 64th Annual meeting ACPA, Bloomfield, Colorado, 2007. Abstract 32. 


\title{
Comentario al trabajo "Tratamiento de la microforma labialı
}

\author{
Dr. Fernando de la Cruz Acosta \\ Cirujano Plástico. Director médico Operación Sonrisa Rep. Dominicana
}

Agradezco a la Directora de la Revista, la confianza depositada en mí para realizar los comentarios del trabajo realizado por el Dr. Luis Monasterio Aljaro, pareciéndome interesantes las aportaciones que hace, sobre todo para corregir el defecto nasal.

Quisiera primero decir con relación al término de microforma labial, que si bien es correcto su uso y que existen diversas clasificaciones y nombres en la literatura médica, la clasificación dada por el Dr. Victor Veau, es quizás la más completa y de fácil comprensión y retención, como lo afirma en su texto Tratamiento del labio leporino y fisura palatina, el Dr. Luis Tresserra Llauradó, resaltando que existe diferencia en lo que sería labio leporino cicatricial (figura 1 izq, $7-$ 8 y 9), donde apenas existe una ligera depresión del borde mucoso asociado a un surco vertical en el labio cutáneo y el labio leporino simple, donde existe una notoria muesca más o menos profunda en el labio superior que abarca todo el bermellón y se extiende hacia todo el labio cutáneo.

En cuanto a la corrección quirúrgica, es cierto que esta condición ha recibido poco interés en la literatura médica, aunque pienso que en la forma cicatricial, su reparación no ofrece dificultad a nivel de lo que sería la línea cutánea, no así en lo que sería el defecto nasal, donde pienso que la aportación del Dr. Monasterio Aljaro es muy apropiada al colocar la incisión superior a nivel del piso nasal y extenderla a la implantación del ala nasal, permitiendo así, como él refiere, abordar el cartílago nasal desplazado, liberarlo de la piel nasal y de la crus medial del cartílago opuesto, y fijándolo con puntos transcolumelares en $\mathrm{U}$; al mismo tiempo este abordaje permite corregir el piso nasal, fijando el músculo transverso nasal a la espina nasal y resolver la diástasis del músculo orbicular.

Decía que en la variedad cicatricial, la corrección del mínimo defecto de la línea cutánea no ofrece dificultad alguna, no así en lo que sería la variedad de labio leporino simple, donde se aprecia la muesca que se puede observar en la Fig. 3 dcha. Pienso que como indicación quirúrgica, la técnica de colgajos triangulares equiláteros (Malek) con sus cuatro variantes, sería la más apropiada para este tipo de defecto simple, ya que se adapta a los diferentes grados de afectación de dicha muesca, aunque también es cierto que la técnica de rotación-avance de Millard también está indicada y ofrece buen resultado, en la variedad cicatricial simple y en otras más complejas, ya que al introducir tejido lateral a la vertiente interna de la fisura labial, corrige la asimetría del piso de la nariz y disimula las suturas.

Pero en esencia, el método de incisiones mínimas que permite resolver las anomalías muscular, nasal y del bermellón, presentado por el Dr. Monasterio Aljaro es muy interesante, sus resultados muy satisfactorios y su planteamiento de que el cirujano debe esforzarse por buscar soluciones a esta anomalía con incisiones mínimas, es muy acertado.

\section{Respuesta al comentario del Dr. Femando de la Gruz Acosta}

\section{Dr. Luis Monasterio Aljaro}

Deseo, en primer lugar, agradecer los comentarios del Dr. Fernando de la Cruz Acosta, en relación al tratamiento de esta patología.

En lo que se refiere a la nomenclatura, en Medicina, es habitual que una misma patología, sea designada con diferentes nombres, lo que contribuye a la confusión, pero el término Microforma, parece ser el más adecuado, para esta forma específica de fisura. La técnica en cuestión, se puede aplicar, además, para casos con fisura labial incompleta, de pequeña cuantía, en los cuales, la fisura, no sea más de un cuarto de la extensión, del labio, con el objeto de reducir la cicatriz visible. Para lesiones mayores se utilizan, como lo señala el Dr. de la Cruz Acosta, técnicas clásicas, aún cuando mi preferencia es por procedimientos rectilíneos tipo Nakayima, Fisher o MillardMoeller, en los cuales, la cicatriz se sitúa sobre la cresta filtral haciéndose inaparente. 